\title{
The Acute Frailty Network - supporting people with frailty and urgent care needs to get home sooner and healthier
}

\author{
Author: Simon Conroy
}

\begin{abstract}
Aims
The Acute Frailty Network (AFN) supports the timely delivery of Comprehensive Geriatric Assessment (CGA) for frail older people in urgent care settings, in order to reduce reliance on acute hospitals, save lives and reduce functional deterioration.
\end{abstract}

\section{Methods}

The AFN supports hospitals in reconfiguring and redesigning services in order to try and optimise the delivery of CGA within each setting. Each site participates for 12 months; the focus of the AFN is on the care of older people with frailty in the first 72 hours of an acute hospital attendance, with an emphasis on early discharge supported by the wider health and social care system. The AFN involves health and social care systems working to improve services locally, supported by national clinical and improvement experts and sharing experiences through national networking events. The Breakthrough Series (BTS) uses a specific quality improvement approach, primarily the Model for Improvement, focusing on Plan-Do-Study-Act cycles to create change. The network team delivers four national events each year, attended by all participating teams, as well as a set of masterclasses and webinars to support teams and enable sharing of experience. Site initiation visits comprise discussion with the site teams about the local context, plans for change and a discussion about possible barriers, as well as a walk-though the patient pathway - from the ED through to 'base wards'. Each participating hospital has an allocated coach to help plan, deliver and measure change.

The evaluation methods include structured telephone interviews and data looking at the processes of both the network and the individual hospitals undertaking improvement work. We report here findings from the first three cohorts that have completed the programme.

\section{Results}

Data on engagement with the AFN is available from the first two cohorts ( 22 hospitals); over $90 \%$ of sites rated the programme as excellent or good, showing high levels of satisfaction with the network, especially site visits, site support, national events to network, measurement support and meetings, and a sense of validation from being part of the AFN. $32 \%$ of hospitals now involve patients and the public in frailty service design. $86 \%$ of sites reported improved partnership working both internally and externally.

In cohort 2, 75\% of sites improved their ability to realise benefits from complex projects (using the NHS Institute's sustainability tool) including areas such as patient benefits, clinical/staff/senior leadership engagement and strategic fit.

Service outcomes from those sites in cohorts 1-3 returning data indicate that $46 \%$ have embedded frailty identification processes, $38 \%$ are delivering CGA within the first 72 hours, 38\% have reduced admissions of frail older people, $54 \%$ have seen reductions in length of stay and $35 \%$ have shown reductions in the number of frail older people in hospital with a length of stay of more than 10 days.

\section{Conclusion}

We have demonstrated that it is possible to run a large scale improvement collaborative aimed at improving urgent care for older people with frailty. A significant proportion of participating sites have been able to demonstrate improvements in key process and outcome metrics.

\section{Conflict of interest statement}

The authors are all employed to work on the Acute Frailty Network. 